\title{
EVOLUTION INTO CONTACT OF THE LOW-MASS CLOSE BINARY SYS- TEMS
}

(Not reviewed)

\author{
M.J. Sarna \\ N. Copernicus Astronomical Center \\ Polish Academy of Sciences \\ 00-716 Warswa \\ Poland
}

\author{
A.V. Fedorova \\ Astronomical Council of the \\ USSR Academy of Sciences \\ Moscow
}

\begin{abstract}
We investigated the effect of mass accretion on the secondary components in close binomy systems $\left(M_{\text {total }} \leq 2.5 M_{\odot} M_{\mathbf{2}, 0} \leq 0.75 M_{\odot}\right)$ exchanging mass in the case $\mathrm{A}$.

The evolution of the low-mass close binary systems $\left(M_{\text {total }} \leq 2.5 M_{\odot}\right)$ exchanging the mass in the case $A$ depends on the three main factors:

- the initial mass ratio $\left(q_{0}=M_{2,0} / M_{1,0}\right)$, which determines the rate of mass transfer between components;

- the inital mass of the secondary component $\left(M_{2,0}\right)$ and

- the effectiveness of the heating of the photosphere of the secondary component, by infalling matter.
\end{abstract}

The second factor allows to divide all systems into two essentially different groups:

a) systems in which the secondary component is a star with a radiative envelope, or with a thin convection zone in the uppermost layers;

b) and systems in which secondary component has a thick convective envelope or is fully convective.

The systems from the first group evolve into contact in a characteristic time scale $10^{5}-10^{7}$ years, and reach contact after transfering of $0.03-0.3 M_{\odot}$. The mass exchange proceeds only in a thermal time scale.

For the systems from the group $b$ the effectiveness of the heating of the stellar surface is the most important. In the case when the entropy of the newly accreted matter is the same as the surface entropy of the secondary, a convective star should shrink upon accretion. Then contact binaries are not formed.

In the case when the entropy of the infalling matter is greater then that on the surface, the reaction of the secondary is different. The radius of the secondary component grows rapidly in response to accretion, and the systems reaches contact after the $10^{3}-310^{6}$ years, and after transfer of $0.002-0.2 . M_{\odot}$. The reaction of the secondary is determined by the formation of the temperature inversion layer below the stellar surface.

Full references in: Sarna, M.J. and Fedorova, A.V. (1988) "Evolutionary status of W UMa-type Binaries - Evolution into contact", Astron. Astrophys., in press. 